\title{
At equilibrium, a fixed-length bidimensional closed string, in tension, built with uniform unit mass along its perimeter, situated in a gravitational field, and separating fluids of different densities, will have a circular shape.
}

Mihail Cocos ( $\square$ mihailcocos@weber.edu )

Weber State University https://orcid.org/0000-0002-3653-4130

Silviu Dorian Chelaru

Weber State University

Research Article

Keywords:

Posted Date: March 8th, 2022

DOI: https://doi.org/10.21203/rs.3.rs-1300186/v1

License: (c) (1) This work is licensed under a Creative Commons Attribution 4.0 International License.

Read Full License 


\title{
At equilibrium, a fixed-length bidimensional closed string, in tension, built with uniform unit mass along its perimeter, situated in a gravitational field, and separating fluids of different densities, will have a circular shape.
}

\author{
Mihail Cocos PhD, Department of Mathematics \\ Weber State University \\ Silviu Dorian Chelaru \\ TRANSOCEANIC LLC - USA (transoceanic.us) \\ Graphics: Architect Felix Perez
}

February 4, 2022

\begin{abstract}
In this paper we calculate the exact shape of a membrane containing a liquid and in dynamic equilibrium. The membrane can be imagined as being the crossection of a cylindrical container immersed in salt water and containing fresh water.
\end{abstract}

\section{Introduction}

At equilibrium, a fixed-length bidimensional closed string, in tension, built with uniform unit mass along its perimeter, situated in a gravitational field, and separating fluids of different densities, will have a circular shape.

This assertion is intended to solve the water needs of many regions of the world by opening a revolutionary mode of marine transportation for fresh water. Yes, fresh water is 
scarce, many times not in the right place, and it is indeed very difficult and costly to transport massive quantities of fresh water at large distances by canals, pipelines, or tankers. Look at Southern California, which despite many wonders of hydraulic engineering works of billions of US\$ still has water shortages. Same problems exist in NW Mexico(Baja), North Africa and the Middle East (NAME), or Australia and South Africa, as well as Western Sahara. The best of the few economical possibilities of long-distance transportation of fresh water is the Ultra-Large Marine Submersible (ULMS) boat that we propose. We call it a boat because that is how submarines are called. Indeed, the ULMS boats are huge submarines that can be hundreds of meters in diameter and thousands of meters in length, built of ballastable reinforced concrete hulls (fresh water is lighter than seawater) that are hollow and filled with millions of tons of fresh water hosted in collapsible bags to be transported over the seas to the destinations of choice. In their transversal round sections, these ULMS boats are the strings we are talking about in our initial assertion. The fact that at equilibrium the ULMS sections hold their circular shape leads to a specific hull construction that has to sustain mainly tension forces, and minimal bending and torsion loads. The complexity of the ULMS boats is given by the fact that their ballasting should be variable (for different states of fresh water contents), should withstand all perturbing forces during cruising, and freshwater loading and unloading, and should also generate no sea ballast water during operation. There is a patent application that solves all these problems (see website transoceanic.us) but in the present document we would concentrate to prove the assertion of our first sentence above: At equilibrium, a fixed-length bidimensional closed string, in tension, built with uniform unit mass along its perimeter, situated in a gravitational field, and separating fluids of different densities, will have a circular shape.

\section{The mathematical problem}

In what follows we will calculate the exact shape of a membrane containing a liquid of density $\rho_{I}$ that is immeresed in a liquid of density $\rho_{E}$ and in dynamic equilibrium. The membrane can be imagined as being the crossection of a cylindrical container immersed in salt water and containing fresh water.

The following are considered to be constant:

- $p_{o}^{I}=$ interior pressure at the origin $O(\mathrm{~kg} / \mathrm{m})$

- $p_{o}^{E}=$ exterior pressure at the origin $O(\mathrm{~kg} / \mathrm{m})$

- $\rho_{I}=$ the density of the interior liquid $\left(\mathrm{kg} / \mathrm{m}^{2}\right)$ 
- $\rho_{E}=$ the density of the exterior liquid $\left(\mathrm{kg} / \mathrm{m}^{2}\right)$

- $g=$ gravitational constant $\left(\mathrm{m} / \mathrm{s}^{2}\right)$

- $m_{u}=$ density of the membrane $(\mathrm{kg} / \mathrm{m})$

- $L=$ length of the membrane $(m)$

Assumptions, notations and figure. The thickness of the membrane is negligible. The membrane cannot be stretched nor compressed in the tangent direction.

We will consider an arc length parametrization of the membrane

$$
x=x(s), y=y(s)
$$

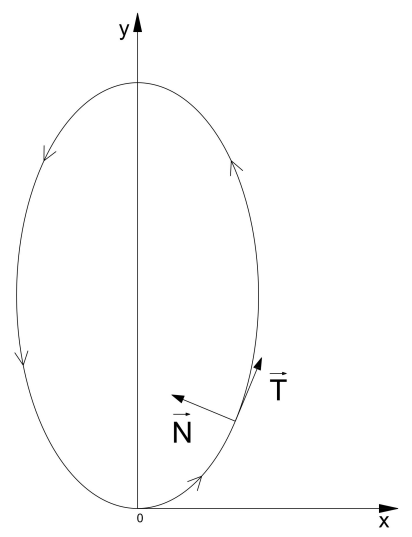

Figure 1: Membrane with chosen orientation

where the arc-length parameter is

$$
0 \leq s \leq L
$$


The unit length tangent vector is

$$
\vec{T}=<x^{\prime}(s), y^{\prime}(s)>
$$

The tangent vector is oriented counterclockwise and we obviously have

$$
|\vec{T}|=\left(x^{\prime}\right)^{2}+\left(y^{\prime}\right)^{2}=1
$$

The unit length vector normal to $\vec{T}$ and pointing towards the interior of the membrane is

$$
\vec{N}=<-y^{\prime}, x^{\prime}>
$$

The curvature equations are

$$
x^{\prime \prime}=-k(s) y^{\prime} \quad ; \quad y^{\prime \prime}=k(s) x^{\prime}
$$

In the vector format the curvature equation is

$$
\frac{d \vec{T}}{d s}=k(s) \vec{N}
$$

In the figure $d s$ is assumed to be an infinitesimal element of the membrane.

The forces acting upon $d s$ are 


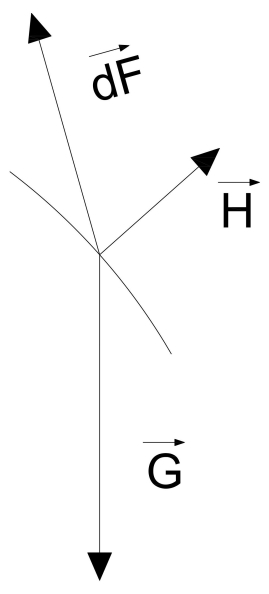

Figure 2: Forces on the membrane

gravity

$$
\vec{G}=<0, m_{u} g d s>
$$

the hydrostatic force $\vec{H}$ acting perpendicular to $d s$

$$
\vec{H}=p(s) d s<y^{\prime},-x^{\prime}>
$$

where $p(s)=p_{o}^{I}-p_{o}^{E}+g y(s)\left(\rho_{I}-\rho_{E}\right)$ at $d s$, and the tension in the membrane 


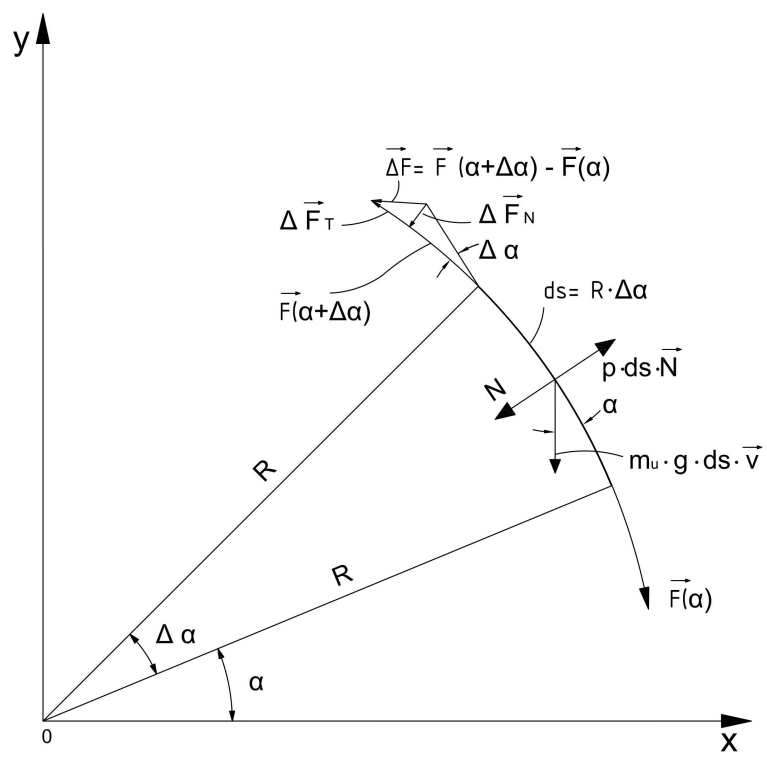

Figure 3: Tension in the membrane

$$
d \vec{F}=\left(\frac{d \vec{F}}{d s} \cdot \vec{T}+\frac{d \vec{F}}{d s} \cdot \vec{N}\right) d s
$$

and since

$$
\vec{F}(s)=f(s) \vec{T}(s)
$$

by differentiating it follows that

$$
d \vec{F}=\left(f^{\prime}(s) \vec{T}(s)+f(s) \frac{d \vec{T}}{d s}\right) d s
$$


Now using (5) we get

$$
d \vec{F}=\left(<f^{\prime}(s) x^{\prime}(s)-f(s) k(s) y^{\prime}(s), f^{\prime}(s) y^{\prime}(s)+f(s) k(s) x^{\prime}(s)>\right) d s
$$

The membrane is in equilibrium iff

$$
\vec{G}+\vec{H}+d \vec{F}=0
$$

Componentwise this translates into

$$
\left\{\begin{array}{l}
p(s) y^{\prime}(s) d s+f^{\prime}(s) x^{\prime}(s d s)-f(s) k(s) y^{\prime}(s) d s=0 \\
m_{u} g d s-p(s) x^{\prime}(s) d s+f^{\prime}(s) y^{\prime}(s) d s+f(s) k(s) x^{\prime}(s) d s=0
\end{array}\right.
$$

Dividing by $d s$ in both equations of the system above we get

$$
\left\{\begin{array}{l}
p(s) y^{\prime}(s)+f^{\prime}(s) x^{\prime}(s)-f(s) k(s) y^{\prime}(s)=0 \\
m_{u} g-p(s) x^{\prime}(s)+f^{\prime}(s) y^{\prime}(s)+f(s) k(s) x^{\prime}(s)=0
\end{array}\right.
$$

After a few algebraic steps, taking into account (2) we solve for $f^{\prime}(s)$ and $f(s) k(s)$ and we get

$$
f^{\prime}(s)=-m_{u} g y^{\prime}(s)
$$

and

$$
k(s) f(s)=p(s)-m_{u} g x^{\prime}(s)
$$

It is relatively easy to verify that (15) together with (16) are equivalent to the component wise equilibrium (14). We will work with (15) together with (16) since they are simpler and relate the magnitude of tension directly to the curvature of the membrane.

\section{Circular membrane}

In what follows we shall verify directly that (15) together with (16) are satisfied in the case of a circle. Since the axis of coordinates are centered at the highest point of the membrane and the depth is measured on the $y$ axis it follow that in the case of a circle we have:

$$
\left\{\begin{array}{l}
x(s)=\frac{L}{2 \pi} \sin \frac{2 \pi s}{L} \\
y(s)=\frac{L}{2 \pi}\left(1-\cos \frac{2 \pi s}{L}\right)
\end{array}\right.
$$

where $0 \leq s \leq L$ 
It follows that

$$
\left\{\begin{array}{l}
x^{\prime}(s)=\cos \frac{2 \pi s}{L} \\
y^{\prime}(s)=\sin \frac{2 \pi s}{L}
\end{array}\right.
$$

and

$$
k(s)=\frac{2 \pi}{L}=\frac{1}{R}
$$

In (19) $R$ is the radius of the circle.

With these explicit values for $x(s), y(s), k(s)$ equations (16) becomes

$$
f(s)=R\left(p(s)-m_{u} g \cos \frac{2 \pi s}{L}\right)
$$

and thus we get a very explicit format for the scalar function $f(s)$. Differentiating (20)we get

$$
f^{\prime}(s)=R\left(g\left(\rho_{I}-\rho_{E}\right)+m_{u} g \frac{2 \pi}{L}\right) \sin \frac{2 \pi s}{L}
$$

and taking into account (15) we obtain

$$
R\left(g\left(\rho_{I}-\rho_{E}\right)+m_{u} g \frac{2 \pi}{L}\right) \sin \frac{2 \pi s}{L}=-m_{u} g \sin \frac{2 \pi s}{L}
$$

Dividing by $\sin \frac{2 \pi s}{L}$ and taking into account that $R \frac{2 \pi}{L}=1$ we get

$$
R g\left(\rho_{I}-\rho_{E}\right)+m_{u} g=-m_{u} g
$$

which gives

$$
R=\frac{2 m_{u}}{\rho_{E}-\rho_{I}}
$$

Please note that the equation (24) is equivalent to the Archimedean equilibrium of the system:

$$
2 \pi R m_{u}+\rho_{I} \pi R^{2}=\rho_{E} \pi R^{2}
$$

Now using equation (16) and the explicit equations of this section we get

$$
f(s)=\left(\frac{2 m_{u}}{\rho_{E}-\rho_{I}}\right)\left(p_{o}^{I}-p_{o}^{E}+g y(s)\left(\rho_{I}-\rho_{E}\right)-m_{u} g \cos \frac{2 \pi s}{L}\right)
$$




\section{Unicity of the contour}

We'd like to show that the two equations (15) and (16) together with the initial conditions

$$
x(0)=0, y(0)=0, x^{\prime}(0)=1, y^{\prime}(0)=0
$$

uniquely determines the contour( i.e the functions $x(s)$ and $y(s)$ ). To do this we will use Picard's theorem on the existence and uniqueness of first order systems of ODE's. We will however apply it to the pair of functions $y(s)$ and $\theta(s)$, where the function $\theta(s)$ is defined by the equations

$$
\left\{\begin{array}{l}
x^{\prime}(s)=\cos \theta(s) \\
y^{\prime}(s)=\sin \theta(s)
\end{array}\right.
$$

Please note that $\theta(s)$ is well defined by using equation (2). Let's multiply equation (16) on both sides by $y^{\prime}(s)$. We get

$$
k(s) y^{\prime}(s) f(s)=p(s) y^{\prime}(s)-m_{u} g x^{\prime}(s) y^{\prime}(s)
$$

and since $k(s) y^{\prime}(s)=-x^{\prime \prime}(s)$ we get

$$
-x^{\prime \prime}(s) f(s)=p(s) y^{\prime}(s)-m_{u} g x^{\prime}(s) y^{\prime}(s)
$$

Now using the chain rule and (28) we get $x^{\prime \prime}(s)=-\theta^{\prime}(s) \sin \theta(s)$ and replacing it into (30) we get

$$
\theta^{\prime}(s)(\sin \theta(s)) f(s)=p(s) y^{\prime}(s)-m_{u} g x^{\prime}(s) y^{\prime}(s)
$$

Integrating equation (15) we get

$$
f(s)=c-m_{u} g y(s)
$$

with $c$ an arbitrary constant. Putting (31) and (32) together we get

$$
\theta^{\prime}(s)(\sin \theta(s))\left(c-m_{u} g y(s)\right)=p(s) y^{\prime}(s)-m_{u} g x^{\prime}(s) y^{\prime}(s)
$$

and taking into account that $y^{\prime}(s)=\sin \theta(s)$ and $x^{\prime}(s)=\cos \theta(s)$ we get

$$
\theta^{\prime}(s)(\sin \theta(s))\left(c-m_{u} g y(s)\right)=p(s) \sin \theta(s)-m_{u} g \cos \theta(s) \sin \theta(s)
$$

Finally, dividing by $\sin \theta(s)$ we get

$$
\theta^{\prime}(s)\left(c-m_{u} g y(s)\right)=p(s)-m_{u} g \cos \theta(s)
$$


Now let us choose $c$ so that

$$
c>m_{u} g L
$$

It follows that we can divide by $\left(c-m_{u} g y(s)\right)$ and the equation (37) becomes

$$
\theta^{\prime}(s)=\frac{p(s)-m_{u} g \cos \theta(s)}{\left(c-m_{u} g y(s)\right)}
$$

Taking into account that $y^{\prime}=\sin \theta(s)$ and that $p(s)=p_{o}^{I}-p_{o}^{E}+g y(s)\left(\rho_{I}-\rho_{E}\right)$ we obtain a first order system of differential equations in the unknowns $\theta(s), y(s)$ explicitly as

$$
\left\{\begin{array}{l}
\theta^{\prime}(s)=\frac{p_{o}^{I}-p_{o}^{E}+g y(s)\left(\rho_{I}-\rho_{E}\right)-m_{u} g \cos \theta(s)}{\left(c-m_{u} g y(s)\right)} \\
y^{\prime}(s)=\sin \theta(s) \\
y(0)=0 \\
\theta(0)=0
\end{array}\right.
$$

The system (38) satisfies the conditions of Picard Theorem and thus prove that the solution is unique provided that the constant $c$ is unique. Here we have to point out that the uniqueness of the constant $c$ follows from the fact that the solution is a contour( i.e. $x(0)=x(L)$ and $y(0)=y(L)$ ) and therefore the system (38) with the added boundary conditions becomes overdetermined

$$
\left\{\begin{array}{l}
\theta^{\prime}(s)=\frac{p_{o}^{I}-p_{o}^{E}+g y(s)\left(\rho_{I}-\rho_{E}\right)-m_{u} g \cos \theta(s)}{\left(c-m_{u} g y(s)\right)} \\
y^{\prime}(s)=\sin \theta(s) \\
y(0)=0=y(L) \\
\theta(0)=0 \\
\theta(L)=2 \pi
\end{array}\right.
$$

and thus $c$ is uniquely determined by the constants $p_{o}^{I}, p_{o}^{E}, \rho_{I}, \rho_{E}, g$, and $m_{u}$.

\section{Final remarks}

As mentioned above the configuration of ULMS fresh water transportation systems has been covered by the patent application shown on our website transoceanic.us . The feasibility of the ULMS systems is also analyzed on an appended simulation sheet that shows the characteristics and the financial parameters of the said systems for specific fresh water transport applications. We covered an international ULMS system for nine billion metric tons (nine cubic kilometers) per year with supply from southern Mexico (Balsas River) and delivery to the region of the Lower Colorado River. The cost of transportation (with 
no profit and no cost of water included) is about $\$ 0.07 /$ metric ton (cubic meter), which is about $5 \%$ of the cost of desalination. The fresh water quantities proposed to be transported by this system are very large, being more than five times the quantity of water that enters Mexico from the Colorado River and almost half of the total US/Mexico water allocation from this river system. The cost of this ULMS system is estimated at US $\$ 8$ billion, which is reasonable taking into account that it is configured to deliver water quantities sufficient for the whole urban population of Southern California, Arizona, and NW Mexico (for about 30 million people). Of course, smaller as well as larger ULMS systems can be built, depending on the specific needs for fresh water and its availability. It is expected that after a slow initial implementation of the ULMS concept, the ULMS systems will proliferate and have an important impact upon the economies and politics of many countries and regions. The missing technical link in massive water transportation and import-export is solved by the ULMS systems, which can transfer gigatonnes of low-cost river water to arid lands, turning them into gardens, feeding the WORLD with bountiful crops, getting plentiful water to cities, and capturing/arresting $\mathrm{CO} 2$ into the carbon-poor soil and new plants. The TRANSOCEANIC systems are meant to lead to peace and cooperation in the world. 